\title{
Mechanisms of Directional Polymer Crystallization
}

\author{
Alejandro A. Krauskopf, Andrew M. Jimenez, Elizabeth A. Lewis, Bryan D. Vogt, Alejandro J. Müller, \\ and Sanat K. Kumar*
}

Cite This: ACS Macro Lett. 2020, 9, 1007-1012

Read Online

\section{ACCESS \\ Џlll Metrics \& More \\ Article Recommendations \\ Supporting Information}

ABSTRACT: Zone annealing, a directional crystallization technique originally used for the purification of semiconductors, is applied here to crystalline polymers. Tight control over the final lamellar orientation and thickness of semicrystalline polymers can be obtained by directionally solidifying the material under optimal conditions. It has previously been postulated by Lovinger and Gryte that, at steady state, the crystal growth rate of a polymer undergoing zone annealing is equal to the velocity at which the sample is drawn through the temperature gradient. These researchers further implied that

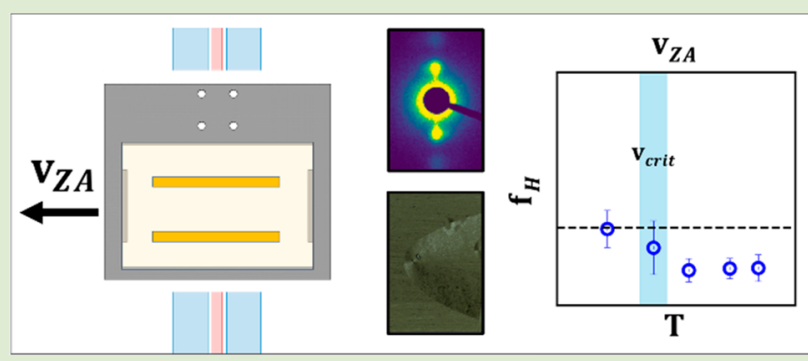
directional crystallization only occurs below a critical velocity, when crystal growth rate dominates over nucleation. Here, we perform an analysis of small-angle X-ray scattering, differential scanning calorimetry, and cross-polarized optical microscopy of zone-annealed poly(ethylene oxide) to examine these conjectures. Our long period data validate the steady-state ansatz, while an analysis of Herman's orientation function confirms the existence of a transitional region around a critical velocity, $v_{\text {crit }}$ where there is a coexistence of oriented and isotropic domains. Below $v_{\text {crit }}$ directional crystallization is achieved, while above $v_{\text {crit }}$ the mechanism more closely resembles that of conventional isotropic isothermal crystallization.

\begin{abstract}
A promising technique that has found extensive use in semiconductor processing is zone annealing (ZA). In this methodology, a sample is subjected to a moving temperature gradient that induces melting, followed by the directional solidification of the material and the accompanying segregation of impurities to the sample extremities. Metallurgists have extensively utilized ZA to produce very pure semiconductors. ${ }^{1,2}$ The directional morphology induced by the crystal front results in anisotropic properties, which have been exploited in applications. These concepts associated with ZA have been applied to both amorphous block copolymer ${ }^{3-8}$ and semicrystalline polymer systems. ${ }^{9-17}$

Nearly 50 years ago, Lovinger and Gryte (L\&G) were one of the first to have applied ZA to polymers using semicrystalline poly(ethylene oxide) (PEO); ${ }^{9,10,18}$ this technique was later applied to isotactic polypropylene, ${ }^{11}$ even-even polyamides, ${ }^{12,13}$ and poly(vinylidene fluoride). ${ }^{14}$ L\&G postulated that the effective directional crystallization of polymers only occurs when the crystal growth rate, $G$, exceeds the nucleation rate, $N$. In other situations, when the nucleation rate is high, the sample typically crystallizes isotropically due to radial growth around the large number of primary nuclei. The question, then, is what determines the $G$ and $N$ and the conditions under which directional crystallization occurs. From in situ microscopy during ZA, L\&G confirmed that the solidmelt interface remained stationary in a moving frame of reference. ${ }^{19}$ This allowed them to propose that under steadystate conditions the growth rate, $G$, must equal the rate at
\end{abstract}

which the heat source moves relative to the sample. This conjecture of steady-state operation, while logical, has not been proven to date. Further, this ansatz implies that the translation rate can be used to map the ZA protocol onto an effective isothermal crystallization process with the same $G$. We term this effective isothermal crystallization temperature, which has the same $G$, as $T_{c \text {,eff. }}$ From this effective isothermal crystallization temperature, the nucleation rate per unit volume in the sample follows $\int_{T_{\mathrm{m}}}^{T_{c, \text { eff }}} N(T) \mathrm{d} T$, where $N(T)$ is the instantaneous nucleation rate at temperature $T$. This past work raises two questions that are assumed to be correct but are unproven: (i) How reasonable is it to map the ZA protocol onto an isothermal crystallization process at $T_{\mathrm{c}, \mathrm{eff}}$ ? (ii) How do we locate the transition from isotropic to directional growth as the velocity of the moving source is varied systematically? In this work, we address these two underpinning questions by conducting detailed studies of PEO crystallized in the presence of a moving heat source.

The ZA setup was modeled after the design of Singh et al. ${ }^{6}$ The temperature gradient was generated by sandwiching a hot

Received: May 4, 2020

Accepted: June 17, 2020

Published: June 23, 2020 
A

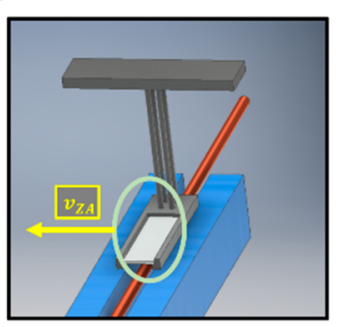

B

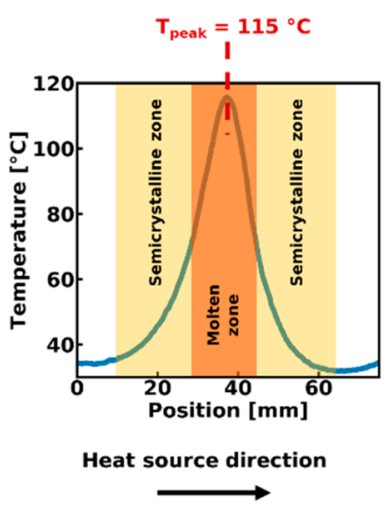

C
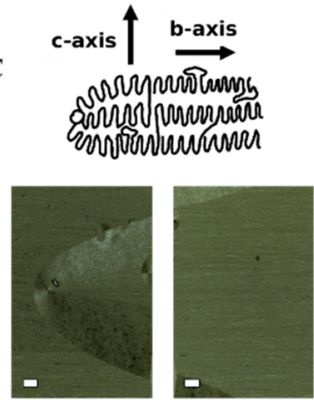

Pulling direction
D

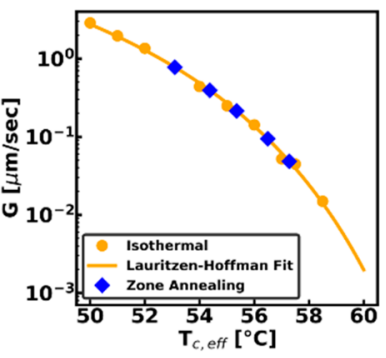

Figure 1. (A) Zone-annealing setup (AutoDesk Inventor rendering). (B) Temperature gradient profile on a sample holder. (C) Cross-polarized optical microscopy of zone-annealed PEO and a schematic of the proposed mechanism of crystallization. Scale bars are $100 \mu \mathrm{m}$. (D) Isothermal crystal growth rate data from polarized light optical microscopy ${ }^{20}$ and Lauritzen-Hoffman analysis.

A

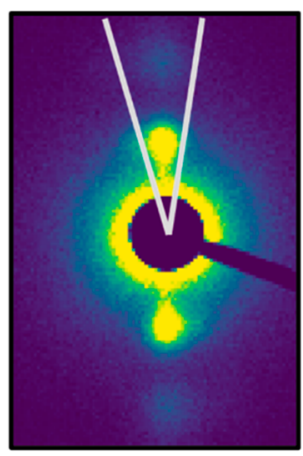

C

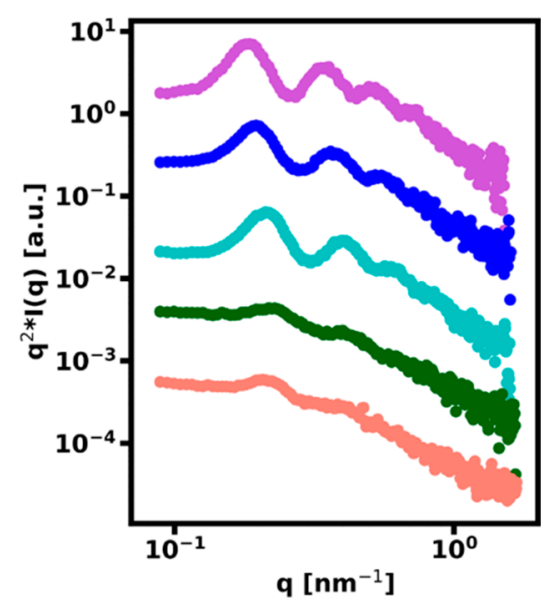

D

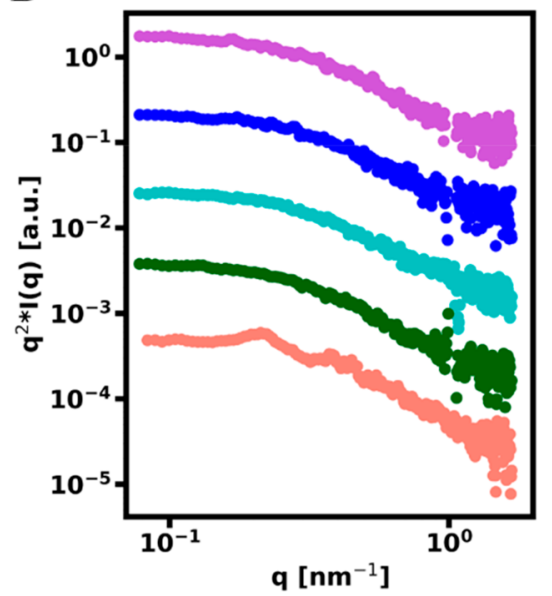

$\mathbf{E}$

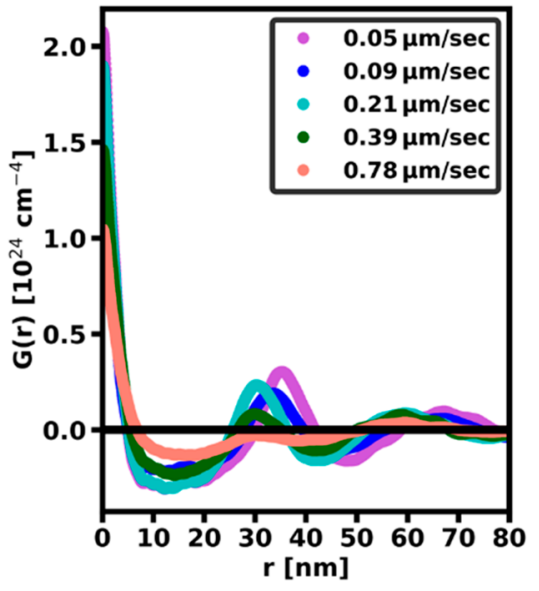

F

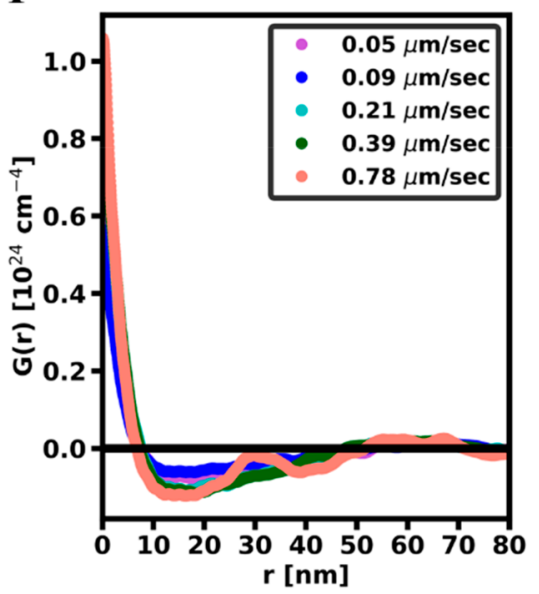

Figure 2. SAXS data obtained on crystallized PEO samples employing the ZA method described in Figure 1. (A) Representative 2D scattering pattern $\left(v_{\mathrm{ZA}}=0.05 \mu \mathrm{m} / \mathrm{s}\right)$ showing wedge integration of $25^{\circ}$ centered on the angle of maximum intensity and (B) $25^{\circ}$ centered orthogonal to the angle of maximum intensity. (C) Representative Lorentz-corrected SAXS profiles for wedge integration around angle of maximum intensity and (D) orthogonal to the angle of maximum intensity. (E) Corresponding correlation functions for angle of maximum intensity and (F) orthogonal to angle of maximum intensity.

resistance wire between two water-chilled cold plates (Figure 1A) at $34{ }^{\circ} \mathrm{C}$. This produces a peak temperature of $115{ }^{\circ} \mathrm{C}$. The maximum gradient, $d T / d x$, was $8{ }^{\circ} \mathrm{C} / \mathrm{mm}$. This gradient produced a molten zone at its maximum of approximately $35 \%$ of the sample length (Figure 1B), while laterally the temperature remained relatively constant with minor edge 
A

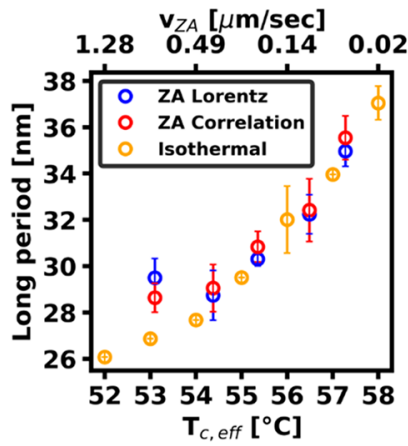

B

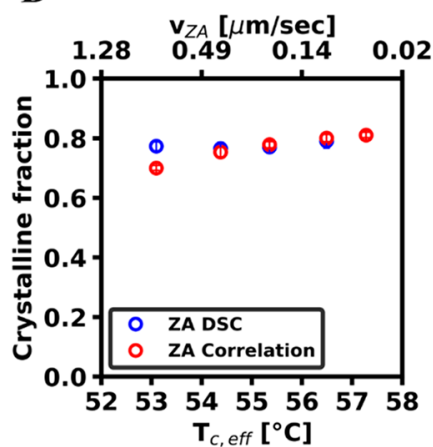

C

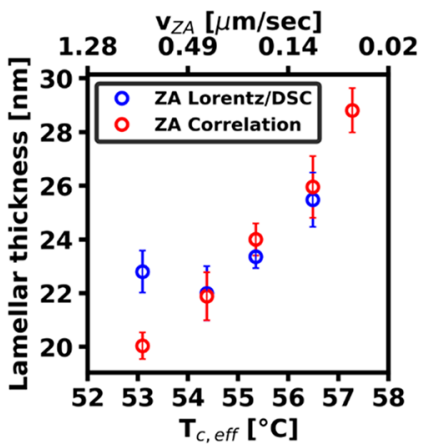

Figure 3. (A) Long period, with $L=\frac{2 \pi}{q^{*}}$, from Lorentz-corrected profiles for zone annealing (blue) and isothermally crystallized samples (orange); the position of the first maximum for the correlation function derived from the zone annealing samples (red) is also plotted. (B) Bulk crystalline volume fraction $\varphi_{c, v}$ from DSC (blue) and linear crystallinity $w_{\mathrm{c}}=\frac{1+\sqrt{1-\frac{4 r_{0}}{L}}}{2}$ from the correlation function for zone annealing (red). $r_{0}$ is the position where the correlation function first crosses zero. (C) Lamellar thickness, calculated as $L \times \varphi_{c, v}$, from the Lorentz-corrected profiles and DSC (blue) and $L \times w_{c}$ from the correlation function for zone annealing (red).

effects. The sample holder was translated at a constant velocity, $v_{\mathrm{ZA}}$ over this heat source. PEO $\left(M_{\mathrm{w}}=100 \mathrm{~kg} / \mathrm{mol}, M_{\mathrm{w}} / M_{\mathrm{n}} \sim\right.$ 4) was used in our experiments. The samples were unconfined rectangular films between two quartz slides such that they could undergo changes in the lateral and pulling directions, but there was no appreciable change in sample geometry after ZA. The detailed protocol for ZA is in the SI.

The isothermal crystal growth rates of PEO were determined by tracking the rate of spherulitic growth using cross-polarized optical microscopy (CPOM). In particular, we used the published data of Jimenez et al. ${ }^{20}$ for a variety of PEO systems. To compare to ZA samples, these growth rates were fit to $\mathrm{L}$ a u ritze $-\mathrm{H}$ off m a n theory: ${ }^{21}$ $G=G_{0} \exp \left(-\frac{U^{*}}{R\left(T_{c}-T_{\infty}\right)}\right) \exp \left(-\frac{K_{\mathrm{g}}}{T_{f} \Delta T}\right)$ where $G_{0}$ is a preexponential constant; $U^{*}$ is an activation energy characteristic of the transport of polymer segments across the melt-crystal front $\left(6280 \mathrm{~J} \mathrm{~mol}^{-1}\right) ; R$ is the gas constant $\left(8.314 \mathrm{~J} \mathrm{~mol}^{-1} \mathrm{~K}^{-1}\right) ; T_{\infty}$ is $T_{\mathrm{g}}-30(178.15 \mathrm{~K}) ; T_{\mathrm{g}}$ is the glass transition temperature; $K_{\mathrm{g}}$ is the rate of surface nucleation; $f$ is a factor defined as $f=\frac{2 T_{\mathrm{c}}}{T_{\mathrm{m}}^{\infty}+T_{\mathrm{c}}}$ that corrects for the temperature dependence of the heat of fusion; $\Delta T=T_{\mathrm{m}}^{0}-T_{\mathrm{c}}$; and $T_{\mathrm{m}}^{0}$ is the equilibrium melting temperature $(352.15 \mathrm{~K}) .^{22}$ For simplicity, all parameters were assumed to be constant except for $T_{\mathcal{c}} \Delta T$, $K_{g}$, and $f$. A linear fit of $\ln (G)$ vs $\frac{1}{T f \Delta T}$ was used to interpolate the $G$ vs $T_{c}$ data. These data provide the $T_{c, \text { eff }}$ corresponding to each growth rate, $G$, assuming that the $L \& G$ ansatz works (see Figure 1D). However, it is unclear if the other structural characteristics of the ZA samples (i.e., long period, crystallinity, crystal thickness) also map to the isothermally crystallized samples. That is, we ask if a ZA sample takes on the same crystalline properties as a sample isothermally crystallized at $T_{c, \text { eff }}$ or not.

To this end, we characterize samples processed with these two techniques with differential scanning calorimetry (DSC) and small-angle X-ray scattering (SAXS). Before obtaining structural parameters, note that ZA samples are anisotropic scatterers, so that performing a full azimuthal integration of the 2D SAXS patterns is inappropriate (see Figure 2A for the scattering pattern of a sample crystallized at $v_{\mathrm{ZA}}=0.05 \mu \mathrm{m} / \mathrm{s}$ ). To address this anisotropy, a wedge integration of $25^{\circ}$ centered on the angle of maximum intensity (Figure 2A) was used. The corresponding orthogonal wedge integration (Figure 2B) was also performed to delineate the visually clear anisotropy in the scattering patterns. The Lorentz-corrected scattering profiles for the maximum intensity and orthogonal wedge integrations are presented in Figures 2C and 2D, respectively.

The first-order scattering peak in Figure 2C was fit to extract the peak scattering vector, $q^{*}$, from which the long period, $L$, was calculated as $L=\frac{2 \pi}{q^{*}}$. The same procedure was used for the $2 \mathrm{D}$ patterns from the isothermal experiments, except that the full azimuthal average was used due to the isotropic scattering. Figure 3A illustrates the long period from SAXS for the ZA (blue) and isothermally (orange symbols) crystallized PEO. It is immediately clear that above $T_{c, \text { eff }} \approx 54{ }^{\circ} \mathrm{C}$, the long periods for both ZA and isothermal crystallization virtually coincide. Thus, at this level, the equivalence between an effective isothermal temperature and the $\mathrm{ZA}$ velocity, $v_{\mathrm{ZA}}$ holds. Below $T_{c, \text { eff }} \approx 54{ }^{\circ} \mathrm{C}$, this equivalence breaks down, which possibly indicates that somewhere in this range is a critical velocity, $v_{\text {crit }}$ above which the directional process is no longer equivalent to the corresponding isothermal crystallization process. Again, this was previously postulated by $\mathrm{L} \& \mathrm{G} ;{ }^{9}$ the phenomenon of a critical velocity has also been reported for directional crystallization of nickel and copper. $^{23,24}$

The bulk crystalline fraction is measured via DSC. The integral under the heat flow curve during the first heating cycle provides the enthalpy of fusion, which when normalized by the enthalpy for $100 \%$ crystalline PEO $(205.4 \mathrm{~J} / \mathrm{g})^{25}$ yields $\varphi_{\mathrm{c}, \mathrm{w}}$, the crystal weight fraction. ${ }^{20}$ The crystal volume fraction, $\varphi_{\mathrm{c}, \mathrm{v}}$, is then obtained from the relation ${ }^{26} \varphi_{\mathrm{c}, \mathrm{v}}=\frac{\varphi_{\mathrm{c}, \mathrm{w}}}{\varphi_{\mathrm{c}, \mathrm{w}}+\left(\rho_{\mathrm{c}} / \rho_{\mathrm{a}}\right)\left(1-\varphi_{\mathrm{c}, \mathrm{w}}\right)}$, where $\rho_{\mathrm{c}}$ and $\rho_{\mathrm{a}}$ are the crystalline ${ }^{27}$ and amorphous ${ }^{28}$ densities, respectively. It should be noted that the ZA samples were cooled to room temperature before heating in the DSC. The heating scans included crystals formed during the cooling process, while the isothermal samples were heated in the DSC directly from $T_{c}$. Therefore, the heating scans included crystals formed at that temperature only. Additionally, errors in the determination of the crystalline fraction are typically in the $10-15 \%$ range. These factors contribute to the fact that the 
Table 1. Structural Parameters from SAXS and DSC of ZA Samples

$\begin{array}{lccccc}v_{\mathrm{ZA}}[\mu \mathrm{m} / \mathrm{s}] & 0.05 & 0.09 & 0.21 & 0.39 & 0.78 \\ T_{c, \text { eff }}\left[{ }^{\circ} \mathrm{C}\right] & 57.3 & 56.5 & 55.4 & 54.4 & 53.1 \\ L[\mathrm{~nm}]^{a} & 35.0 \pm 0.7 & 32.2 \pm 0.8 & 30.3 \pm 0.3 & 28.7 \pm 1.1 & 29.5 \pm 0.8 \\ L[\mathrm{~nm}]^{b} & 35.5 \pm 0.9 & 32.4 \pm 1.4 & 30.8 \pm 0.7 & 29.1 \pm 1.0 & 28.6 \pm 0.6 \\ \varphi_{c, v}^{c} & & 0.81 \pm 0.03 & 0.79 \pm 0.01 & 0.78 \pm 0.02 & 0.79 \pm 0.02 \\ w_{\mathrm{c}}{ }^{b} & 0.81 \pm 0.01 & 0.80 \pm 0.01 & 0.78 \pm 0.01 & 0.75 \pm 0.02 & 0.70 \pm 0.01 \\ l_{\mathrm{c}}{ }^{a, c} & & 26.0 \pm 1.1 & 23.9 \pm 0.5 & 22.5 \pm 1.1 & 23.3 \pm 0.8 \\ l_{\mathrm{c}}{ }^{b} & 28.8 \pm 0.8 & 26.0 \pm 1.2 & 24.0 \pm 0.6 & 21.9 \pm 0.9 & 20.0 \pm 0.5\end{array}$

${ }^{a}$ From Lorentz-corrected scattering profiles ${ }^{b}$ From the correlation function ${ }^{c}$ From DSC.

\section{A}

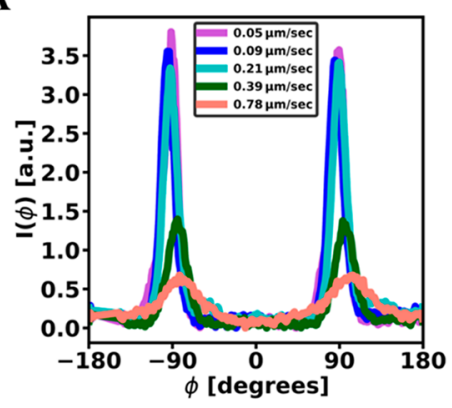

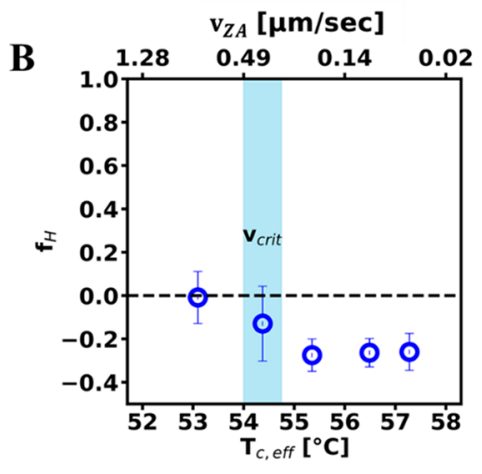

B

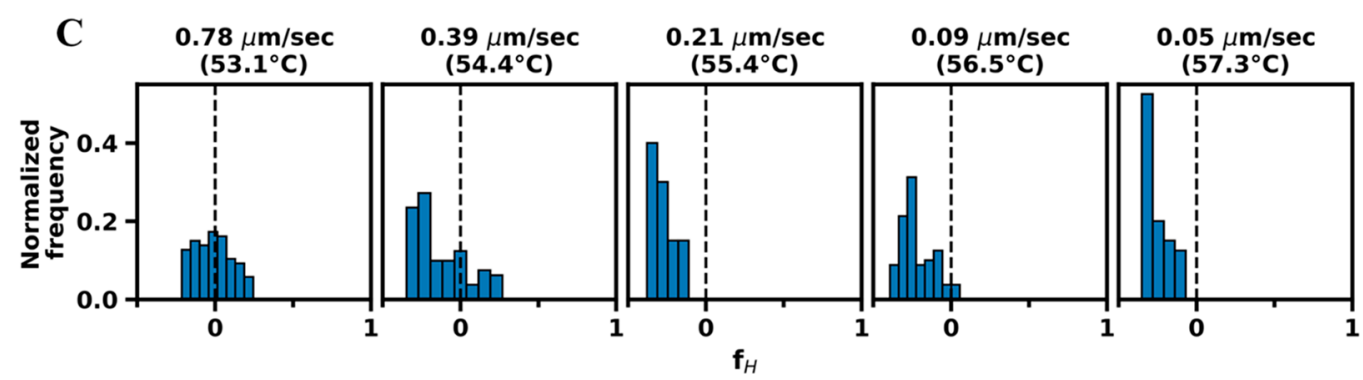

Figure 4. (A) Representative intensity profiles as a function of azimuthal angle, $\phi$, for all probed $v_{\mathrm{ZA}} . \phi=0^{\circ}$ corresponds to the pulling direction. (B) Average Herman's orientation function for zone-annealed PEO as a function of $T_{c, \text { eff }}$ (C) Normalized distributions of Herman's orientation function measured across the area of the sample.

$\varphi_{\mathrm{c}, \mathrm{v}}$ values are not comparable across the crystallization methods. We therefore only present the zone annealing $\varphi_{c, v}$ in Figure 3B (blue); the full data set is shown in the SI. The crystal lamellar thickness follows naturally as $l=L^{*} \varphi_{c, v}$; these values are plotted in blue for ZA in Figure 3C.

To independently verify the structural parameters from SAXS and DSC, the correlation function for each scattering profile was calculated and analyzed. This procedure was originally published by Strobl and Schneider ${ }^{29}$ and later used by other researchers ${ }^{30-32}$ (details can be found in the SI). In short, the long period, linear crystallinity, and crystal thickness can be extracted under the assumption of a periodic, two-phase model. Representative correlation function profiles for each $v_{\mathrm{ZA}}$, calculated from the wedge integration centered on the angle of maximum intensity, are shown in Figure 2E. The corresponding correlation function profiles for the orthogonal integrations are presented in Figure 2F.

The calculation of the correlation function typically requires extrapolation of the intensity data to both $q=0$ and $q \rightarrow \infty$. Throughout our analysis, it was found that only the invariant was affected significantly by incorporating the extrapolated data into the integral; the structural parameters studied here were not affected appreciably. However, for completeness, the extrapolation to both extremes was performed (see SI).
The structural parameters extracted from SAXS, DSC, and the correlation function analysis are listed in Table 1 and shown in Figure $3 .{ }^{33}$ These structural parameters are consistent between the analysis methods, thus validating the two methods employed. The deviation of the lamellar thickness for the fastest $v_{\mathrm{ZA}}$ (lowest $T_{\mathrm{c}, \text { eff }}$ ) can be explained by the DSC crystallinity being a bulk measurement, while the correlation function linear crystallinity is a localized value for a region of the sample with high anisotropy. We speculate that this might reflect the fact that isotropic (maybe nonisothermal) crystallization processes are competing with the directional crystallization process at the fastest $v_{\mathrm{ZA}}$.

Given that we probed velocities around $v_{\text {crit }}$ it is instructional to examine the scattering anisotropy as in Figure 2A to further elucidate the characteristics of this critical velocity. Parenthetically, we note that the X-ray spot sizes here are 200 $\mu \mathrm{m}$. The intensities as a function of the azimuthal angle $\phi$ are plotted in Figure 4A. The extent of anisotropy is quantified using Herman's orientation function, which is the ensemble average of the second Legendre polynomial: ${ }^{34}$ $f_{\mathrm{H}}=\frac{3\left\langle\cos ^{2}(\phi)\right\rangle-1}{2}$ where $\left\langle\cos ^{2}(\phi)\right\rangle=\frac{\int_{0^{\circ}}^{180^{\circ}} I(\phi) \sin (\phi) \cos ^{2}(\phi) \mathrm{d} \phi}{\int_{0^{\circ}}^{180^{\circ}} I(\phi) \sin (\phi) \mathrm{d} \phi}$. Note that we carry out the integration to $180^{\circ}$ instead of $90^{\circ}$ due to the 2 -fold symmetry of our SAXS patterns. The 
reference angle in our system, $\phi=0^{\circ}$, is chosen to be the direction associated with $v_{\mathrm{ZA}}$. When $f_{\mathrm{H}}=0$, the scatterers are isotropically distributed; when $f_{\mathrm{H}}=1$, the intensity is purely along the reference ZA direction; and when $f_{\mathrm{H}}=-0.5$, the intensity is solely perpendicular to the reference angle direction (see SI for calculations of $f_{\mathrm{H}}$ for these extremes). Values in between these extremes indicate intermediate orientation. For each $v_{\mathrm{ZA}}$, 42-87 scattering patterns on different spots were collected over 2-4 samples, and $f_{\mathrm{H}}$ was calculated for each spot. The average $f_{\mathrm{H}}$ for each $v_{\mathrm{ZA}}$ is plotted in Figure $4 \mathrm{~B}$; the normalized distributions of $f_{\mathrm{H}}$ for each $v_{\mathrm{ZA}}$ are presented in Figure $4 \mathrm{C}$. For the fastest $v_{\mathrm{ZA}}$, i.e., for the lowest $T_{\mathrm{c}, \text { eff }}$, the $f_{\mathrm{H}}$ is zero, within error, indicating that the lamellae have no statistical preference in their direction of crystallization relative to the ZA direction. This again confirms that the polymer chains are crystallizing isotropically at this $v_{\mathrm{ZA}}$. The uniform distribution of $f_{\mathrm{H}}$ about zero for this fastest $v_{\mathrm{ZA}}$ further proves this point.

Moving to slower $v_{\mathrm{ZA}}$ (i.e., higher $T_{\mathrm{c}, \mathrm{eff}}$ ), the distribution shifts to more negative values, indicating that the crystal correlations are perpendicular to the pulling direction; this can be seen in the $2 \mathrm{D}$ pattern in Figure $2 \mathrm{~A}$ and in the additional SAXS patterns provided in the SI. At $v_{\mathrm{ZA}}=0.39 \mu \mathrm{m} / \mathrm{s}$, there are two populations of $f_{\mathrm{H}}$ : one is effectively isotropic $\left(f_{\mathrm{H}}=0\right)$, and the other is directional $\left(f_{\mathrm{H}}<0\right)$. This is the critical velocity, $v_{\text {crit }}$, below which $f_{\mathrm{H}}$ shifts primarily to negative values.

Before concluding, it is appropriate to compare this protocol with another one which results in oriented morphologies, i.e., flow-induced crystallization. In the latter case, it is now wellestablished that flow-induced precursors, e.g., those created by the orientation of long chains, lead to directed crystallization. ${ }^{35}$ As expected, these phenomena manifest themselves at large flow rates where long chains themselves orient. Our results are opposite in spirit, namely, that directed crystallization preferentially occurs at low velocities; in contrast, large velocities yield isotropic orientations (Figure 4). We estimate the time scale for the flow to convect a chain its own size to be $\tau_{\text {flow }} \sim b \sqrt{N} / v$, where $N$ is the chain length and $b$ is the Kuhn length of the chains in question. For the chain lengths used and the critical velocity, this corresponds to $\tau_{\text {flow }} \sim 0.1 \mathrm{~s}$. This is comparable to the relaxation time of the chains at a temperature of $373 \mathrm{~K}$ obtained from the rheological crossover of $G^{\prime}$ and $G^{\prime \prime}$, the storage and loss moduli of the viscoelastic response, respectively (unpublished data from our laboratory). So, for smaller velocities, the chains are able to fully relax, and thus they are not likely to be distorted by the flow. Indeed, the chain orientation that is deduced based on our results (schematic Figure 1C), i.e., normal to the velocity (or temperature gradient) direction, clearly argues against any flow-induced chain orientation effects. An analogous case, that of transcrystallization, also originates from molecularly oriented precursors, typically fibrillar fillers which template the polymer matrix. ${ }^{36}$ Thus, some other mechanism must be operative, and we believe that the L\&G conjecture that the dominance of crystal growth over nucleation, accompanied by the presence of a temperature gradient, plays a central role in the orientation obtained.

In this work, we have established the validity of the steadystate conjecture for the zone crystallization of polymers, allowing direct mapping of the structural parameters of ZA samples to isothermally crystallized ones. We also verify the existence of a critical velocity, $v_{\text {crit }}$, which delineates the transition between nominally oriented crystals and isotropic crystals, from the distributions of Herman's orientation function. Taken in conjunction, these discoveries should allow us to exert greater control over the final lamellar orientation and crystal thickness of semicrystalline polymers. Mechanistically, below $v_{\text {crit }}$ the $b$-axis of the polymer chain orients preferentially along the pulling direction (Figure 1C), while above $v_{\text {crit, }}$ this order is absent. This transition is not abrupt since intermediate velocities show the coexistence of order and disorder. These data provide evidence for the validity of previously proposed directional crystallization mechanisms of Asano et al. for crystallization of polyethylene under a moving temperature gradient, ${ }^{15}$ as well as $L \& G$ for poly(ethylene oxide). ${ }^{10}$ Finally, although not discussed extensively, we find that the geometry of the sample (confined in a tube vs unconfined film) does not impact the mechanism.

\section{ASSOCIATED CONTENT}

\section{Supporting Information}

The Supporting Information is available free of charge at https://pubs.acs.org/doi/10.1021/acsmacrolett.0c00346.

Experimental methods, discussion of Lauritzen-Hoffman analysis, extrapolation of SAXS data and correlation function analysis, comparison of correlation functions calculated with and without extrapolation of SAXS data, Herman's orientation function calculation for delta function intensities, additional SAXS 2D scattering patterns, additional CPOM micrographs, DSC heat flow curves, and isothermal DSC and crystal lamellar thickness data set (PDF)

\section{AUTHOR INFORMATION}

\section{Corresponding Author}

Sanat K. Kumar - Department of Chemical Engineering, Columbia University, New York, New York 10027, United States; ○ orcid.org/0000-0002-6690-2221; Email: sk2794@ columbia.edu

\section{Authors}

Alejandro A. Krauskopf - Department of Chemical Engineering, Columbia University, New York, New York 10027, United States; ○ orcid.org/0000-0002-9070-0427

Andrew M. Jimenez - Department of Chemical Engineering, Columbia University, New York, New York 10027, United States; 이이. orcid.org/0000-0001-7696-9705

Elizabeth A. Lewis - Department of Polymer Engineering, University of Akron, Akron, Ohio 44325, United States

Bryan D. Vogt - Department of Chemical Engineering, The Pennsylvania State University, University Park, Pennsylvania 16803, United States; 이이.org/0000-0003-1916-7145

Alejandro J. Müller - Ikerbasque, Basque Science Foundation, 48011 Bilbao, Spain; POLYMAT and Department of Polymer Science and Technology, Faculty of Chemistry, Basque Country University UPV/EHU, 20018 Donostia-San Sebastián, Spain

Complete contact information is available at:

https://pubs.acs.org/10.1021/acsmacrolett.0c00346

\section{Author Contributions}

The manuscript was written through contributions of all authors. All authors have given approval to the final version of the manuscript.

\section{Notes}

The authors declare no competing financial interest. 


\section{ACKNOWLEDGMENTS}

This work was supported by grants DE-SC0018182, DESC0018135, and DE-SC0018111, funded by the U.S. Department of Energy, Office of Science. A.A.K. acknowledges funding from the Gates Millennium Scholars program under Grant No. OPP1202023 from the Bill \& Melinda Gates Foundation. A.J.M. acknowledges funding from the Basque Government through grant IT1309-19. We are grateful to Dr. Andrew J. Lovinger for critically reviewing the manuscript and offering multiple comments that have helped us to significantly improve our paper. We would also like to thank Beatrice Bellini for experimental assistance.

\section{REFERENCES}

(1) Pfann, W. G. Zone Melting. Science (Washington, DC, U. S.) 1962, 135 (3509), 1101-1109.

(2) Tiller, W. A.; Jackson, K. A.; Rutter, J. W.; Chalmers, B. The Redistribution of Solute Atoms during the Solidification of Metals. Acta Metall. 1953, 1 (4), 428-437.

(3) Majewski, P. W.; Yager, K. G. Millisecond Ordering of Block Copolymer Films via Photothermal Gradients. ACS Nano 2015, 9 (4), 3896-3906.

(4) Samant, S.; Strzalka, J.; Yager, K. G.; Kisslinger, K.; Grolman, D.; Basutkar, M.; Salunke, N.; Singh, G.; Berry, B.; Karim, A. Ordering Pathway of Block Copolymers under Dynamic Thermal Gradients Studied by in Situ GISAXS. Macromolecules 2016, 49 (22), 86338642.

(5) Mita, K.; Takenaka, M.; Hasegawa, H.; Hashimoto, T. Cylindrical Domains of Block Copolymers Developed via Ordering under Moving Temperature Gradient: Real-Space Analysis. Macromolecules 2008, 41 (22), 8789-8799.

(6) Singh, G.; Yager, K. G.; Smilgies, D. M.; Kulkarni, M. M.; Bucknall, D. G.; Karim, A. Tuning Molecular Relaxation for Vertical Orientation in Cylindrical Block Copolymer Films via Sharp Dynamic Zone Annealing. Macromolecules 2012, 45 (17), 7107-7117.

(7) Singh, G.; Batra, S.; Zhang, R.; Yuan, H.; Yager, K. G.; Cakmak, M.; Berry, B.; Karim, A. Large-Scale Roll-to-Roll Fabrication of Vertically Oriented Block Copolymer Thin Films. ACS Nano 2013, 7 (6), 5291-5299.

(8) Xue, J.; Singh, G.; Qiang, Z.; Yager, K. G.; Karim, A.; Vogt, B. D. Facile Control of Long Range Orientation in Mesoporous Carbon Films with Thermal Zone Annealing Velocity. Nanoscale 2013, 5 (24), 12440-12447.

(9) Lovinger, A. J.; Gryte, C. C. The Morphology of Directionally Solidified Poly(Ethylene Oxide) Spherulites. Macromolecules 1976, 9 (2), 247-253.

(10) Lovinger, A. J.; Lau, C. M.; Gryte, C. C. Microstructure of Directionally Solidified Poly(Ethylene Oxide). Polymer 1976, 17 (7), 581-586.

(11) Lovinger, A. J.; Chua, J.; Gryte, C. C. Studies on the $\alpha$ and $\beta$ Forms of Isotactic Polypropylene by Crystallization in a Temperature Gradient. J. Polym. Sci., Polym. Phys. Ed. 1977, 15, 641-656.

(12) Lovinger, A. J. Crystallographic Factors Affecting the Structure of Polymeric Spherulites. I. Morphology of Directionally Solidified Polyamides. J. Appl. Phys. 1978, 49, 5003.

(13) Lovinger, A. J. Crystallographic Factors Affecting the Structure of Polymeric Spherulites. II. X-Ray Diffraction Analysis of Directionally Solidified Polyamides and General Conclusions. J. Appl. Phys. 1978, 49, 5014.

(14) Lovinger, A. J.; Wang, T. T. Investigation of the Properties of Directionally Solidified Poly(Vinylidene Fluoride). Polymer 1979, 20, $725-732$.

(15) Asano, T.; Mina, F.; Nishida, A.; Yoshida, S.; Fujiwara, Y. Crystallization of a Low Molecular Weight Polyethylene and Paraffins Under A Temperature Gradient. J. Macromol. Sci., Part B: Phys. 2001, B40, 355-367.
(16) Ye, C.; Zhang, L.; Fu, G.; Karim, A.; Kyu, T.; Briseno, A. L.; Vogt, B. D. Controlled Directional Crystallization of Oligothiophenes Using Zone Annealing of Preseeded Thin Films. ACS Appl. Mater. Interfaces 2015, 7 (41), 23008-23014.

(17) Ye, C.; Wang, C.; Wang, J.; Wiener, C. G.; Xia, X.; Cheng, S. Z. D.; Li, R.; Yager, K. G.; Fukuto, M.; Vogt, B. D. Rapid Assessment of Crystal Orientation in Semi-Crystalline Polymer Films Using Rotational Zone Annealing and Impact of Orientation on Mechanical Properties. Soft Matter 2017, 13 (39), 7074-7084.

(18) Lovinger, A. J.; Gryte, C. C. Model for the Shape of Polymer Spherulites Formed in a Temperature Gradient. J. Appl. Phys. 1976, 47 (5), 1999-2004.

(19) Lovinger, A. J.; Chua, J.; Gryte, C. C. An Apparatus for in Situ Microscopy of Zone Solidifying Polymers. J. Phys. E: Sci. Instrum. 1976, 9, 927.

(20) Jimenez, A. M.; Krauskopf, A. A.; Pérez-Camargo, R. A.; Zhao, D.; Pribyl, J.; Jestin, J.; Benicewicz, B. C.; Müller, A. J.; Kumar, S. K. Effects of Hairy Nanoparticles on Polymer Crystallization Kinetics. Macromolecules 2019, 52, 9186-9198.

(21) Hoffman, J. D.; Miller, R. L. Kinetics of Crystallization from the Melt and Chain Folding in Polyethylene Fractions Revisited: Theory and Experiment. Polymer 1997, 38 (13), 3151-3212.

(22) Wu, L.; Lisowski, M.; Talibuddin, S.; Runt, J. Crystallization of Poly(Ethylene Oxide) and Melt-Miscible PEO Blends. Macromolecules 1999, 32 (5), 1576-1581.

(23) Baker, I.; Li, J. Directional Annealing of Cold-Rolled Copper Single Crystals. Acta Mater. 2002, 50, 805-813.

(24) Li, J.; Johns, S. L.; Iliescu, B. M.; Frost, H. J.; Baker, I. The Effect of Hot Zone Velocity and Temperature Gradient on the Directional Recrystallization of Polycrystalline Nickel. Acta Mater. 2002, 50, 4491-4497.

(25) Mark, J. E. Physical Properties of Polymers Handbook; 2007.

(26) Lorenzo, A. T.; Arnal, M. L.; Albuerne, J.; Müller, A. J. DSC Isothermal Polymer Crystallization Kinetics Measurements and the Use of the Avrami Equation to Fit the Data: Guidelines to Avoid Common Problems. Polym. Test. 2007, 26 (2), 222-231.

(27) Wunderlich, B. Macromolecular Physics; 1973. DOI: 10.1016/ B978-0-12-765601-4.50007-X.

(28) Zhu, L.; Cheng, S. Z. D.; Calhoun, B. H.; Ge, Q.; Quirk, R. P.; Thomas, E. L.; Hsiao, B. S.; Yeh, F.; Lotz, B. Phase Structures and Morphologies Determined by Self-Organization, Vitrification, and Crystallization: Confined Crystallization in an Ordered Lamellar Phase of PEO-b-PS Diblock Copolymer. Polymer 2001, 42, 58295839.

(29) Strobl, G. R.; Schneider, M. Direct Evaluation of the Electron Density Correlation Function of Partially Crystalline Polymers. J. Polym. Sci., Polym. Phys. Ed. 1980, 18, 1343-1359.

(30) Russell, T. P.; Ito, H.; Wignall, G. D. Neutron and X-Ray Scattering Studies on Semicrystalline Polymer Blends. Macromolecules 1988, 21 (6), 1703-1709.

(31) Talibuddin, S.; Wu, L.; Runt, J.; Lin, J. S. Microstructure of Melt-Miscible. Macromolecules 1996, 29 (23), 7527-7535.

(32) Fragiadakis, D.; Runt, J. Microstructure and Dynamics of Semicrystalline Poly(Ethylene Oxide)-Poly(Vinyl Acetate) Blends. Macromolecules 2010, 43, 1028-1034.

(33) There is one missing crystallinity value as we do not have access to the laboratory to complete this measurement.

(34) White, J. L.; Spruiell, J. E. The Specification of Orientation and Its Development in Polymer Processing. Polym. Eng. Sci. 1983, 23 (5), 247-256.

(35) Somani, R. H.; Yang, L.; Zhu, L.; Hsiao, B. S. Flow-Induced Shish-Kebab Precursor Structures in Entangled Polymer Melts. Polymer 2005, 46 (20), 8587-8623.

(36) Dufresne, A.; Kellerhals, M. B.; Witholt, B. Transcrystallization in Mcl-PHAs/Cellulose Whiskers Composites. Macromolecules 1999, 32, 7396-7401. 